\title{
Keith Wrightson
}

\section{Zwei Wege zur Erfassung der englischen Sozialstruktur des 16. und 17. Jahrhunderts ${ }^{*}$}

Die Frage nach der Beschaffenheit der Sozialstruktur ist für die englische Sozialgeschichte des 16. und 17. Jahrhunderts eines der am meisten behandelten Probleme. Das Problem gehört aber auch zu denjenigen, die noch am wenigsten geklärt sind. Daß die englische Gesellschaft in dieser Zeit stark geschichtet war, wird allgemein anerkannt. Veränderungen in der sozialen Verteilung von Grund und Boden, Reichtum, Ansehen und Macht gehören weiterhin zu den am intensivsten diskutierten Themen der Geschichtswissenschaft. Die Debatte über die Sozialstruktur bleibt jedoch oft seltsam oberflächlich und bruchstückhaft. Eine knappe Darstellung der traditionellen sozialen Gliederung bleibt eine Pflichtübung für die Lehrbuchautoren. Nur wenige kommen darüber hinaus. Wohl gibt es Untersuchungen, darunter ausgezeichnete ${ }^{1}$, einzelner sozialer Gruppen, aber keine einzige Arbeit, die sich den Problemen widmet, die die Frage nach der Sozialstruktur insgesamt aufwirft. Die neuere Sozialgeschichte hat sich mehr denn je damit befaßt, schichtspezifische Analysen zu liefern. Charakter und Bedeutung der gesamtgesellschaftlichen Struktur werden jedoch wenig diskutiert und bleiben überraschend undeutlich.

Wie dem auch sei, man entkommt dem Problem nicht. Wie Peter Laslett in seinem einflußreichen (und umstrittenen) Deutungsversuch der englischen Geschichte des 17. Jahrhunderts als Einklassengesellschaft ${ }^{2}$ bemerkte, ist für jegliche zufriedenstellende Diskussion der Schicksale von einzelnen Personen und von Gruppen, für jegliche sinnvolle Annāherung an die Entwicklung der Gesellschaft insgesamt die Klärung des Charakters der Sozialstruktur eine wesentliche Voraussetzung. Ich will in diesem Beitrag versuchen, die Diskussion zu zentrieren, indem ich zwei Wege der Annäherung an das Problem der Sozialstruktur untersuche. Einige Historiker haben sich im wesentlichen darauf konzentriert, zeitgenössiscbe Sebweisen der Sozialstruktur zu erläutern, hauptsächlich, indem sie zeitgenössische Beschreibungen der Sozialordnung diskutieren. Andere beschreiten einen Weg, den man sozialstrukturell nennen kann: Sie

\footnotetext{
- Úbersetzt aus dem Englischen von Fritz Seeberger (Bochum).

' S. z. B. Lawrence Stone, The Crisis of the Aristocracy, 1558-1641 (Oxford 1965); Gordon E. Mingay, The Gentry. The Rise and Fall of a Ruling Class (London 1976); Mildred Campbell, The English Yeoman Under Elizabeth and the Early Stuarts (New Haven 1942); Alan Everitt, Farm Labourers, in: J. Thirsk (Hg.), The Agrarian History of England and Wales IV, 1500-1640 (Cambridge 1967).

${ }^{2}$ Peter Laslett, The World we have lost (London 1965) Kap. 2.
} 
versuchen, die relative Größe der sozialen Gruppen, so wie die Zeitgenossen sie unterschieden haben, zu bestimmen, die soziale Verteilung von Grund und Boden, Einkommen, Ansehen oder Macht zu erhellen und die Breite sozialer Unterschiede in zahllosen Aspekten von Verhalten und Erfahrung aufzudecken. Diese beiden Wege schließen sich gegenseitig natürlich nicht aus, sind aber auch nicht erschöpfend. Eine wirklich angemessene Behandlung der Sozialstruktur würde eine Berücksichtigung beider Wege erfordern - und zusätzlich eine Diskussion der charakteristischen Beziehungsmuster zwischen Individuen und Gruppen mit unterschiedlichen Positionen in der sozialen Hierarchie. Die beiden genannten Wege bieten jedoch einen Anfang, eine vorläufige, beschreibende Analyse der englischen Gesellschaft. Wenn wir sie nacheinander verfolgen, werden wir ein schärferes Bild des gegenwärtigen Forschungsstandes entwickeln, herausragende Probleme erkennen und mögliche Ansätze zukünftiger Untersuchungen und Interpretationen aufspüren.

\section{Die Diskussion zeitgenössischer Auffassungen von Gesellschaft}

In ihren ausgefeiltesten Konzeptionen der Natur des Universums haben Autoren des 16. und 17. Jahrhunderts eine "Great chain of Being" entworfen, die von den Engeln bis zu den Elementen selbst hinabreichte, in der alles Erschaffene seinen ihm zugewiesenen Platz einnahm, und in dem die Aufrechterhaltung der gottgegebenen Ordnung wesentlich war für das harmonische Funktionieren des Ganzen. Nach der offiziellen "Homily on Obedience“ (1547), die regelmāßig in den Pfarrkirchen verlesen wurde, „hat Gott der Allmāchtige alle Dinge im Himmel und auf Erden geschaffen und eingerichtet in höchst gelungener und vollkommener Ordnung. Im Himmel hat er unterschiedliche Ränge und Stufen von Erzengeln und Engeln errichtet. Auf Erden hat er Könige bestimmt und Prinzen, samt den anderen Herrschenden unter ihnen, alles in guter und erforderlicher Ordnung ... Jedem Stand der Menschen sind gemäß seiner Berufung, seiner Würde und seines Amtes Pflichten und Aufgaben zugeteilt. Manche haben einen hohen Rang, manche einen niedrigen; manche sind Könige und Prinzen, manche Untergebene und Untertanen, Priester und Laien, Herren und Diener, Väter und Kinder, Ehemānner und Ehefrauen, reich und arm ... Wo nicht die rechte Ordnung herrscht, da regiert Mißwirtschaft, Fleischeslust, Frevel, Sünde und babylonische Verwirrung." ${ }^{3}$

Die traditionelle Theorie sah also die menschliche Gesellschaft, wie die Natur, als eine Zusammensetzung von funktional miteinander verknüpften, aber ungleichen Teilen - die wechselweise als "degrees“, „orders“ oder „estates“ bezeichnet wurden. Für Sir Thomas Elyot, der während der Herrschaft Heinrichs VIII. schrieb, war die Gesellschaft ein ,aus unterschiedlichen estates und degrees der Menschen zusammen-

${ }^{3}$ Wieder abgedruckt in Geoffrey $\mathrm{R}$. Elton $(\mathrm{Hg}$ ), The Tudor Constitution. Documents and Commentary (Cambridge 1962) $15 \mathrm{f}$. 
gefügter oder geschaffener ... Körper“. Für Robert Burton war sie 1621 bestimmt durch „eine Ungleichheit von states, orders und degrees“4. Aber was genau waren die „degrees“, „orders“ und „estates“, die von den Zeitgenossen gesehen wurden, und wie waren sie definiert? Bei der Beantwortung dieser Frage können wir uns einer großen Anzahl von Beschreibungen der englischen Gesellschaft zuwenden, die weniger ausgefeilt sind und gleichzeitig Besonderheiten berücksichtigen. Das reicht von den sozialen Kategorien, wie sie in der Luxus-Gesetzgebung des 16. Jahrhunderts zur Regelung von Bekleidungsfragen unterschieden wurden, über die Berichte von Autoren wie William Harrison, Sir Thomas Smith, Sir Thomas Wilson und Edward Chamberlayne bis zu den Bemühungen der frühen Sozialstatistik des späten 17. Jahrhunderts, insbesondere Gregory Kingss. Diese Beschreibungen der Gesellschaft unterscheiden sich hinsichtlich ihrer Zwecke und durch unterschiedliche Berücksichtigung jeweiliger Details. Jede hat ihre Eigentümlichkeiten. Es ist jedoch erkennbar, daß sie die gleiche Gesellschaft beschreiben, und ihre kleineren Abweichungen voneinander verleihen dem Gesamtbild, das sich ergibt, eher mehr als weniger Glaubwürdigkeit.

Zunächst: die gebildeten Zeitgenossen beschrieben die Gesellschaft nicht mit Hilfe der drei funktionalen „Stānde“ mittelalterlicher Sozialtheorie, sondern als eine einzige Hierarchie von Status- und Berufsgruppen. Sicherlich blieben einige Elemente des älteren Schemas erhalten. Selbst nach der Reformation wurde der Klerus gelegentlich neben den weltlichen Ständen gesondert behandelt. Die Bezeichnungen „orders" und „estates" wurden häufig benutzt. Dennoch wiederholten die Autoren des 16. und 17. Jahrhunderts in ihren eigenen Beschreibungen der Gesellschaft gewöhnlich nicht die traditionelle Dreiteilung ${ }^{6}$. Sie beschrieben eine einzige Hierarchie von „degrees“,

4 Zitiert bei Perez Zagorin, The Court and the Country. The Beginnings of the English Revolution (London 1969) $23 \mathrm{f}$.

${ }^{3}$ Für nützliche allgemeine Uberblicke über diese Quellen s. N. B. Harte, State Control of Dress and Social Change in Pre-Industrial England, in: Donald C. Coleman/A. H. Jobn (Hg.), Trade, Government and Economy in Pre-Industrial England. Essays presented to F. J. Fisher (London 1976); David Cressy, Describing the Social Order of Elizabethan and Stuart England, in: Literature and History 3 (1976).

'Lateinische und französische Beispiele der „Ständeliteratur" waren in England spätestens seit dem 12.Jahrhundert bekannt. Englischsprachige Beispiele für dieses Genre gibt es seit dem 14. Jahrhundert. Bis zum 15.Jahrhundert waren die „estates“ und "degrees“, die solche Arbeiten auflisteten, jedoch beträchtlich ausgearbeitet worden, als das Genre sich einer verānderten sozialen Wirklichkeit anpaßte. Als Caxton im spāten 15.Jahrhundert von den drei Ständen schrieb, von "clergye, chevalrye and labourers", von "clerkes, knyghtes and labourers“, da war seine schlichte Dreiteilung bereits deutlich konservativ und anachronistisch (wahrscheinlich durch ein Modell aus dem 13. Jahrhundert beeinflußt). Die meisten Autoren verzichteten auf die drei „estates" oder „orders" zugunsten einer größeren Anzahl von „estates" oder „degrees“, die eine einzige Folge bildeten. Im 16.Jahrhundert wurde dies allgemein gebräuchlich. Aber obwohl die ursprünglich drei Stände in eine komplexere Einteilung eingeflossen waren, blieb das Wesentliche der Philosophie einer ,ständischen Welt" lange sehr lebendig: die Betonung gottgegebener Pflichten und funktionaler Abhängigkeiten. Für eine Diskussion der Entwicklung der moralisierenden Ständeliteratur in England (obgleich weniger aufmerksam gegenüber diesen Veränderungen als möglich) s. Robert Mobl, The Three Estates in Medieval and Renaissance Literature (New York 1933) Kap. 4-5. 
und wenn sie von "orders“ oder "estates" sprachen, dann benutzten sie diese Begriffe als Synonyme für „degrees“. In seiner berühmten Description of England (veröffentlicht 1577) sprach William Harrison von den „degrees of people“ in England und versicherte, daß „wir in England unser Volk gewöhnlich in vier Kategorien (sorts) einteilen“: „Gentlemen“ (eine Kategorie, welche den Hochadel, knights, esquires und „diejenigen, die einfach gentlemen genannt werden“, umfaßte); die „citizens und burgesses“ der Städte; die „yeomen“ des platten Landes; und schließlich diejenigen, die er "the fourth sort of people“ nannte, die "weder Stimme noch Macht im Staate haben, sondern regiert werden müssen und nicht andere regieren sollen" (eine Kategorie, die „husbandmen“, Handwerker, Tagelöhner und Diener umfaßte) ${ }^{7}$. Zwei Generationen später unterschied Robert Reyce in seiner Beschreibung der Grafschaft Suffolk sieben „degrees and callings“, nämlich noblemen, knights, gentlemen, yeomen, townspeople, husbandmen und „die Armen"8. Zusammengenommen liefern solche Beschreibungen das, was wir als „klassische Hierarchie“ sozialer Beschreibung im frühneuzeitlichen England bezeichnen können: Der Adel: knights, esquires und „bloße“ gentlemen; die Berufsstände; Stadtbürger; yeomen; husbandmen; Handwerker; Häusler und Tagelöhner; Diener.

Die Herkunft dieser klassischen Hierarchie sozialer Ränge (offensichtlich handelte es sich um eine Vereinfachung einer komplexeren Wirklichkeit, auf die man sich geeinigt hatte) ist ungewiß. Möglicherweise war die Festschreibung von Titular-Kategorien dem Statute of Additions von 1413 zu verdanken, wonach erstmals Beklagte in einem Prozeß ihren „Estate or Degree or Mystery“ anzugeben hatten. Vielleicht hat sie sich weiter ausgebreitet durch die öffentliche Bekanntmachung von Luxus-Bestimmungen. Die traditionelle hierarchische Zusammenstellung von Status- und Berufsgruppen kann auch beeinflußt worden sein durch Vorstellungen über die himmlische Hierarchie und die „chain of being“, durch klassische Modelle, oder dadurch, daß die verschiedenen Stufen innerhalb jeder der drei mittelalterlichen „Stände“ schlicht zu einer einzigen Rangfolge zusammengestellt wurden (wie in den Ritualen des höfischen Lebens oder in öffentlichen Aufzügen) ${ }^{9}$.

Wo auch immer die Herkunft der klassischen Hierarchie liegt, sicher ist, daß sie nie genau definiert oder als Ganze gesetzlich verankert wurde, und diese Tatsache ist wichtig genug, um als zweites Merkmal zeitgenössischer Gesellschaftsbeschreibung

${ }^{7}$ Frederick J. Furnivall (Hg.), Harrison's Description of England (New Shakespeare Society, 6th series, No. 1, London 1877) $105 \mathrm{ff}$.

${ }^{8}$ Zit. in Zagorin, The Court and the Country, 24.

${ }^{9}$ I Hen. V c.5 (1413); Rodney H. Hilton, Bond Men Made Free. Mediaeval Peasant Movements and the English Rising of 1381 (London 1973) 178f.; F. R. H. Du Boulay, An Age of Ambition. English Society in the Late Middle Ages (London 1970) 70; Harte, State Control of Dress, 136; $E$. M. W. Tillyard, The Elizabethan World Picture (London 1943), bes. Kap. 2 \& 4. Für die Beziehung zwischen städtischen Zeremonien und sozialer Hierarchie s. C. Pbytbian-Adams, Ceremony and the citizen. The communal year at Coventry 1450-1550, in: Peter Clark/Paul Slack (Hg.), Crisis and Order in English Towns 1500-1700 (London 1972) und Mervyn James, Ritual, Drama and the Social Body in the Late Medieval English Town, in: Past and Present 98 (1983). Für eine Diskussion älterer Arbeiten, die sich mit Fragen von Rangfolgen beschäftigen, s. Mobl, The Three Estates, $129 \mathrm{ff}$. 
betont zu werden. Natürlich gab es für einige soziale Gruppen allgemein anerkannte Kriterien der Zugehörigkeit. Der Hochadel der dukes, earls, marquises, viscounts und barons hob sich ab durch den Besitz vererbbarer Titel, eine begünstigte Rechtsposition und den privilegierten Parlamentarierstatus als Mitglieder des Oberhauses. Knights wurden für den Königsdienst ernannt. Andere Adelsränge waren für die königlichen heralds, die regelmäßig die Provinzen besuchten, formal definiert. Trotzdem hatten die Zeitgenossen große Schwierigkeiten, unterhalb der Ebene der knights eindeutige Kriterien für einen sozialen Status anzugeben. Das herausragendste Beispiel solch einer Verwirrung sind die Definitionen von ,gentility“. William Harrison meinte zum Beispiel, unter ,gentlemen“ könnte man zusammenfassend diejenigen verstehen, „die durch ihre Abstammung und ihr Blut oder zumindest durch ihre Tugenden geadelt und bekannt sind“. Das ist schon mehrdeutig genug, aber Harrison sah sich genötigt hinzuzufügen, daß ,gentle status“ sowohl erworben als auch ererbt werden könne, und in der abschließenden Analyse gestand er „gentility“ jedem Mann zu, der „ohne körperliche Arbeit leben und sich Lebensführung, Aufwand und Haltung eines gentleman leisten kann “10. Abgesehen von solchen Schwierigkeiten (und sie tauchten bei dem weniger dringlichen Fall einer Definition von „yeoman“ erneut auf) gab es eine Menge Unsicherheiten, was die zutreffende hierarchische Einordnung bestimmter Berufs- und Sozialgruppen betraf, vor allem bei den Mitgliedern jener Gruppen, die Lawrence Stone als „halb-unabhängige Berufsgruppen“ bezeichnet hat: den niederen Klerus, Kaufleute, Ärzte, Rechtsanwälte und Amtsinhaber ${ }^{11}$. Waren führende Kaufleute und Akademiker gentlemen oder nicht? Sollten sie, was den Status betraf, an die landbesitzende Gentry angeglichen werden oder getrennt einen eigenen, etwas niedrigeren Rang einnehmen? Die zeitgenössischen Autoren waren sich darüber nicht klar, und es gab keine eindeutige Übereinkunft.

Wenn wir diesen Schwierigkeiten weiter nachspüren, stoßen wir auf ein drittes Merkmal dieser Darstellungen der Sozialordnung: die ungeheure Komplexität der Kriterien sozialer Einschätzung und Bewertung. Formal wurde die Sozialstruktur als eine Statushierarchie präsentiert. Aber diejenigen, die sie zu beschreiben versuchten, mußten erkennen, daß „rank“ und „degree“ keine unabhängigen Variablen waren. Definitionsschwierigkeiten stellten sich ein, weil sie sich aus mehreren Merkmalen zusammensetzten. Eine Vielfalt von Kriterien war in unterschiedlichem Ausmaß beteiligt. Dazu gehörten Geburt, ererbter oder verliehener Titel, Reichtum und die Art dieses Reichtums (zum Beispiel Grund und Boden, Handel, akademische Ausbildung oder Lohnarbeit), Beruf, Lebensstil, rechtlicher Status, rechtlicher Charakter des Landbesitzes, die Besetzung von Machtpositionen in der Dorfgemeinde oder auf nationaler Ebene. Das Luxus-Gesetz von 1533 zum Beispiel kannte Kategorien, die auf dem beruhten, was als „komplizierte, ineinander verschachtelte Beziehung“ von Geburt, Titel, Höhe des Einkommens, Beruf, Landbesitz und Lohnarbeit bezeichnet worden ist ${ }^{12}$.

${ }^{10}$ Zum Problem, wie ein gentleman zu definieren sei, s. $R$. Kelso, The Doctrine of the English Gentleman in the sixteenth century (Urbana 1929) Kap. 2.

${ }^{11}$ Lawrence Stone, Social Mobility in England, 1500-1700, in: Past and Present 33 (1966) $18 \mathrm{ff}$.

${ }_{12}$ Harte, State Control of Dress, 136. Für eine vollständigere Diskussion der Kriterien sozialer Rangfolge s. Keith Wrightson, English Society 1580-1680 (London 1982) Kap.1. 
Viele Zeitgenossen sehnten sich nach einer einfachen und unveränderlichen sozialen Ordnung, aber das Sozialsystem, das sie zu beschreiben versuchten, beruhte nicht auf eindeutigen, wohldefinierten, formalen Positionen. Es war kein wasserdicht abgeschottetes Kastensystem, nicht durch Vorstellungen religiöser Reinheit und Unreinheit abgesichert. Es war kein gesetzlich verankertes Standessystem. Die herkömmliche soziale Hierarchie der Zeit war weniger selbst eine Institution als vielmehr ein Nebenprodukt anderer sozialer Institutionen.

Dieser Mangel an Rigidität und klaren Definitionen wird weitgehend bestätigt durch ein viertes Merkmal zeitgenössischer Sozialbeschreibung: die offene, wenn auch gelegentlich neidvolle Anerkennung der Tatsache, daß individuelle soziale Mobilität in der englischen Gesellschaft alltägliche Realität war. Ein Status konnte erreicht und er konnte verloren werden. Sir Thomas Wilson sprach mit Verachtung über die Söhne von yeomen, die eine Ausbildung bekamen und auf der Stelle in Samt und Seide herumliefen, das Gehabe von Landadeligen annahmen und auf der Anrede „Mr.“ bestanden, dem Kennzeichen eines gentleman, wohingegen ihre Väter noch mit Haus- und Vornamen allein ausgekommen waren ${ }^{13}$.

In einer berühmten Passage bemerkte Sir Thomas Smith: „Was den gentleman betrifft, so ist er in England billig zu haben. ${ }^{\text {14 }}$ Es kam darauf an, sich den erforderlichen Lebensstil leisten zu können und von den adligen Nachbarn anerkannt zu werden. Und wenn ein höherer sozialer Status gewonnen werden konnte, so konnte er ebenso wieder verloren werden. William Cecil, Lord Burghley, riet seinem Sohn: „Der gentleman, der einen acre Land verkauft, verliert an Kredit, denn gentleman zu sein heißt, überlieferte Reichtümer zu besitzen. Und wenn das Fundament zu sinken beginnt, dann muß unweigerlich auch das Bauwerk fallen." ${ }^{15}$

Ähnlich glaubte William Harrison, daß der ,gewisse Vorrang und die größere Wertschätzung“, die einen Mann mit dem Status eines yeoman in seinem Dorf kennzeichneten, von der Möglichkeit abhing, „wohlhabend zu leben, einen ansehnlichen Haushalt zu unterhalten und sich für die Erlangung von Reichtümern abzurackern ${ }^{\text {“16. }}$.

Mit mehr oder weniger großer Klarheit sagen die zeitgenössischen Sozialanalytiker, daß es sich um eine stark geschichtete und höchst statusbewußte Gesellschaft handelte, daß aber die "degrees“, die in der sozialen Hierarchie üblicherweise unterschieden wurden, zum größten Teil keinem festgelegten, inneren Aufbau folgten und keine unabhängige, institutionalisierte Realität bildeten. Der relative Status ergab sich jeweils aus dem Zusammenspiel zahlreicher Variablen, und in diesem komplexen Prozeß konnte man einzelnen einen gewissen „Punktestand“ zurechnen, in einem Prozeß größtenteils informeller sozialer Einschätzung, der eine Vielfalt von Kriterien beinhaltete. Zweifellos gab es mit „Vermögen“ eine einzelne, grundlegende Determinante für den Rang. Ohne Vermögen konnte kein Aspirant auf einen höheren Status darauf hoffen, seine Ansprüche durchzusetzen und zu halten. Aber man würde die Realität

${ }^{13}$ Joan ThirskJJobn P. Cooper (Hg.), Seventeenth-century Economic Documents (Oxford 1972) 752.

${ }^{14}$ Zit. in Kelso, Doctrine of the English Gentleman, 26.

${ }^{15}$ Ebd., 28.

${ }^{16}$ Furnivall (Hg.), Harrison's Description of England, 133. 
jener Tage karikieren, wenn man behauptete, daß andere Kriterien, einige offenbar archaischer Natur, nur beiläufige Bedeutung gehabt hätten. Vermögen gehörte notwendigerweise zu einem Status, aber es genügte nicht immer. Das System sozialer Einschätzung beinhaltete, wie die Ansichten und Meinungen des Volkes auch, sowohl eine geistige Anpassung an die sozio-ökonomische Realität als auch „Bruchstücke vieler unterschiedlicher Denksysteme" ${ }^{\text {"17 }}$.

Die Untersuchung zeitgenössischer Gesellschaftsbeschreibungen kann also viel darüber zeigen, wie bestimmte Gebildete der Zeit ihre eigene Gesellschaft wahrnahmen. Sie dient dazu, die Komplexität und gelegentliche Uneindeutigkeit dieser Wahrnehmung zu unterstreichen. Sie ist ein bekannter und wertvoller Weg, sich der Sozialordnung zu nähern. Aber sie hat ihre Schwierigkeiten und ihre Grenzen.

Erstens gibt es das allgemeine Problem, inwieweit man im wesentlichen literarischen Quellen ohne einen unabhängigen Nachweis ihrer Richtigkeit vertrauen kann, wenn es um die Wiedergabe sozialer Realität geht. Zweitens gibt es eine Reihe von speziellen Fragestellungen, die unbeantwortet bleiben, wenn wir uns auf Quellen dieser Art beschränken.

Das naheliegendste Problem ist, daß zeitgenössische Beschreibungen der sozialen Hierarchie nur selten Angaben über den relativen Anteil der Bevölkerung enthalten, der einer bestimmten Kategorie zugeordnet wird. Das aktuelle Profil der Schichtung bleibt im Dunkeln, und das schließt nicht nur den Vergleich mit anderen Gesellschaften aus, sondern auch die Aufdeckung signifikanter regionaler und lokaler Unterschiede innerhalb Englands ${ }^{18}$.

Auch hier liefern diese Quellen im wesentlichen Blicke von oben. Bei den bekanntesten Beispielen des 16. und 17.Jahrhunderts sind die Autoren hauptsāchlich damit beschäftigt, die „political nation“ zu beschreiben. Lang und breit beschäftigen sie sich mit dem Hochadel und der gentry. Bei sozialen Bestimmungen unterhalb der Ebene der yeomanry auf dem Land und der Vollbürger in den Städten tendieren sie dazu, diese zu minimieren oder sie nur am Rande zu behandeln. Wenn wir soziale Ungleichheit unter der Masse der Bevölkerung für den Großteil dieser Zeit erforschen wollen, dann müssen wir über die Zeugnisse hinausgehen, die die Mehrzahl der zeitgenössischen Sozialbeschreibungen liefern ${ }^{19}$.

${ }^{17}$ Die zitierte Passage bei Keith Thomas, Religion and the Decline of Magic. Studies in popular beliefs in sixteenth and seventeenth century England (London 1971) 185.

${ }^{18}$ Natürlich schlossen einige zeitgenössische Beschreibungen Schātzungen der Größe bestimmter sozialer Gruppen ein. Das herausragendste Beispiel ist Gregory Kings oft wiedergegebenes "Scheme of the Income and Expense of the Several Families of England Calculated for the Year 1688“, s. Thirsk/Cooper, Seventeenth-Century Economic Documents, $780 \mathrm{f}$. Aber selbst wenn sie als zuverlässig genommen werden können, liefern solche Zahlen auf nationaler Ebene lediglich Schätzwerte. Für neuere Diskussionen der fraglichen Zuverlässigkeit der Schätzwerte Gregory Kings s. Geoffrey S. Holmes, Gregory King and the Social Structure of Pre-Industrial England, in: Transactions of the Royal Historical Society, 5th series, 27 (1977) und Peter H. Lindert/Jeffrey G. Williamson, Revising England's Social Tables, 1688-1812, in: Explorations in Economic History 19 (1982).

19 Sehr gut zeigt dies Cressy, Describing the social order, 31 . 
Ebenso ernst muß man die Schwierigkeit nehmen, daß diese Wiedergaben der Gesellschaft nicht als völlig vertrauenswürdige Führer zur Art und Weise zeitgenössischer Wahrnehmungen der Sozialordnung betrachtet werden können. Sie sagen uns nichts darüber, wie Angehörige der mittleren und unteren „degrees“ ihre soziale Welt wahrgenommen haben - und es ist möglich, daß deren Wahrnehmungen sich stark von denen gebildeter Gesellschaftskommentatoren unterschieden.

Außerdem erhalten wir aus diesen Beschreibungen ein zum größten Teil statisches, stilisiertes Bild der englischen Gesellschaft. Daraus ergibt sich der Eindruck von Beständigkeit und relativer Stabilität, der in die Irre führen kann. Es sollte nicht vergessen werden, daß die Beschreibungen der abgestuften Hierarchie durch gebildete Zeitgenossen in Werken, die zur Veröffentlichung oder zur Verbreitung bestimmt waren, zum größten Teil eine formale Übung sozialer Klassifikation war. In weniger formalen Augenblicken wurden viel simplere Begriffe verwandt. Die informelle Sprache sozialer Klassifikation, die man aus Quellen wie Briefen, Aussagen vor Gericht, Petitionen und der Pamphlet-Literatur entnehmen kann, unterschied zum Beispiel nur zwischen „gentleman“, „der mittleren Schicht des Volkes“ und der „unteren“ oder „armen Schicht“. Im Zusammenhang mit sozialen Auseinandersetzungen war man auch mit noch gröberen Kategorien schnell genug bei der Hand - die „Menge“, der „Mob“, die „Bauernlümmel“, der „Pöbel“.

All dies deutet auf die weitverbreitete Existenz von einfacheren, gröberen Auffassungen über die grundlegenden Gruppierungen in der Sozialordnung des frühneuzeitlichen Englands hin. In einer neueren Studie über Unruhen in den östlichen Fenlands während der dreißiger Jahre des 17. Jahrhunderts wird an zeitgenössischer Terminologie zitiert: „rich men“; ,great men“; „, man of quality“; „sufficient men“; ,the better sort“; „able persons of good estate“; "the meaner sort“; „the ruder sort"; ,poor labou-

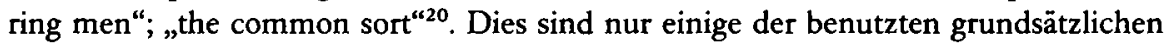
Klassifikationen, und sie enthüllen uns eine Welt sozialer Bedeutungen, die von den meisten formalen Beschreibungen dieser Zeit nicht berührt wird. Die Historiker, die sich mit dem frühneuzeitlichen England beschäftigen, wissen sehr wohl, daß es solch eine alternative Sprache sozialer Beschreibung gibt, und doch werden die Folgerungen daraus nur unbefriedigend erfaßt. Generationen von Studenten sind vertraut gemacht worden mit der klassischen Hierarchie sozialer Analyse. Die informelle Sprache aber, die so häufig und kraftvoll vom Volk benutzt wurde, wartet noch immer auf eine wissenschaftliche Untersuchung. Bis diese Arbeit erledigt ist, kann nicht wirklich zu Recht behauptet werden, wir hätten bereits ein völlig ausreichendes Verständnis davon, wie die Menschen des 16. und 17.Jahrhunderts ihre Gesellschaft erfaßten und wie sich diese Auffassungen im Laufe der Zeit möglicherweise verānderten. Wir können nur sagen, daß es außer der anerkannten, überkommenen Art der Beschreibung sozialer Ordnung auch Alternativen gab. Darüber hinaus können wir zu Recht vermuten, daß die gröberen Auffassungen, wie sie in dem, was man als Sprache der "sorts" be-

${ }^{20}$ Die zitierten Beispiele stammen aus Keith Lindley, Fenland Riots and the English Revolution (London 1982). 
zeichnen könnte, verkörpert sind, im Alltag von größerer praktischer Bedeutung waren. Tatsächlich nahm ihre Verwendung zu. Zu Beginn des 18. Jahrhunderts war diese alternative Redeweise so dominant geworden, daß sie das Sprechen von „degrees“ ersetzte. Der naturalisierte Schweizer Guy Miège, der eine der populärsten Darstellungen der englischen Gesellschaft schrieb, verkörpert diesen Übergang. Zu Anfang schrieb er noch von „orders and degrees", aber nachdem er die nobility and die gentry behandelt hat, verwendet er die Begriffe "middle sort", "common sort", „,inferior sort" und „meaner sort“21.

Aus all diesen Gründen sollten die englischen Historiker ausgesprochen vorsichtig sein, bevor sie formale Darstellungen der sozialen Hierarchie für bare Münze nehmen oder ihnen vorrangige Bedeutung bei der Erklärung des Charakters der Sozialordnung im 16. und 17.Jahrhundert beimessen. Wenn wir das Risiko vermeiden wollen, den Beschränkungen verhaftet zu bleiben, die durch parteiliche und nachweislich irreführende Bilder dieser Gesellschaft vorgegeben sind, dann müssen diese Quellen durch unabhāngige Belege überprüft und ergänzt werden. Solche unabhängigen Belege sind insbesondere auf dem von mir so genannten sozialstrukturellen Weg der Annäherung an die Analyse der Gesellschaftsordnung zusammengetragen worden.

\section{Der sozialstrukturelle Ansatz}

In den letzten Jahren haben viele Historiker des frühneuzeitlichen Englands quantitative und vergleichende Analysen bestimmter Aspekte der sozialen Schichtung versucht. In ihren Tabellen haben sie gewöhnlich die traditionellen „Stände“ unterschieden, die wir aus der klassischen Hierarchie zeitgenössischer Gesellschaftsbeschreibungen kennen. Durch diese Untersuchungen sind unsere Kenntnisse über die Fakten sozialer Schichtung in dieser Zeit enorm erweitert worden. In einem kurzen Überblick wie diesem hātte es wenig Zweck, die Einzelergebnisse solcher Studien aufzuzählen, die weit über die Literatur verstreut sind und komplexe Probleme der Quellenauswertung und der Methodologie aufwerfen. Es wird jedoch von Nutzen sein, einige der Wege hervorzuheben, über die sie das in den literarischen Quellen präsentierte Gesellschaftsbild bestātigen, klären oder erweitern.

Zunāchst: die sozialstrukturellen Untersuchungen haben gezeigt, daß die traditionelle Gliederung nach Status und Berufsgruppe ohne jeden Zweifel tatsächliche - und in der Tat auch statistisch nachweisbare - Unterschiede einschloß in der Verteilung von Grund und Boden, von Einkommen, Unterschiede im Zugang zu Positionen von Macht und Autorität - genau die Unterscheidungsmerkmale, die auch von zeitgenössischen Gesellschaftsanalytikern am häufigsten betont wurden. Die überragende Stellung des Hochadels und der gentry als eine über Reichtum, Ansehen und Macht verfügende Elite sowie die interne Differenzierung der gentry (in knights, esquires und

21 Wieder abgedruckt in Daniel $A$. Baugb $(\mathrm{Hg}$.), Aristocratic Government and Society in Eighteenth-Century England. The Foundations of Stability (New York 1975) 42, 45, 47, 48. 
„bloße“ gentlemen) sind überreich bestätigt worden ${ }^{22}$. Auch die besondere Stellung der yeomanry ist bestätigt worden ${ }^{23}$. In Dorfgesellschaften verstehen wir jetzt die Unterscheidung zwischen yeomen und husbandmen, zwischen husbandmen und cottagers, zwischen cottagers und landlosen Arbeitern besser. Dies sind Unterscheidungen, die sich in den Verzeichnissen des Landbesitzes, in den Besitzinventaren und in der Verteilung von Gemeindeämtern nur zu deutlich niederschlagen ${ }^{24}$. Für die städtische Gesellschaft haben Untersuchungen der sozialen Ursprünge führender Kaufleute, Hāndler und Akademiker uns geholfen, die Unklarheiten zu erklären und zu beseitigen, die zeitgenössische Autoren in bezug auf die genaue soziale Stellung dieser Gruppen hatten, vor allem, indem die Enge der Verbindung zwischen städtischen und ländlichen führenden Personen aufgezeigt wurde ${ }^{25}$. Obgleich der Charakter der Schichtung in der städtischen Gesellschaft in mancher Hinsicht undeutlicher bleibt als in der ländlichen, hat es einige erfolgreiche Bemühungen nicht nur zur Rekonstruktion der Verteilung von Reichtum gegeben, sondern auch zur Bestimmung des relativen Ranges bestimmter Gewerbe und Berufe in der Stadt. Wir verzeichnen eine wachsende Aufmerksamkeit für die Unterschiede an Reichtum, an Ansehen und be-

${ }^{22}$ Die besten allgemeinen Arbeiten zum Hochadel und zur gentry sind Stone, Crisis of the Aristocracy und Mingay, The Gentry. Eine ausgezeichnete neuere Zusammenfassung der anhaltenden Debatte über Strukturverānderungen des Landbesitzes bei $C . G$. A. Clay, Economic Expansion and Social Change: England 1500-1700, 2 Bde. (Cambridge 1984), Bd.1, Kap.5. Aus den vielen Studien über landbesitzende Eliten einzelner Grafschaften sind die wertvollsten $J . T$. Cliffe, The Yorkshire Gentry from the Reformation to the Civil War (London 1969) und B. G. Blackwood, The Lancashire Gentry and the Great Rebellion (Chetham Society, 3rd series, Bd. 25 1978). Eine Diskussion methodologischer Probleme bei der Beschäftigung mit der landgesessenen gentry bei Jobn S. Morrill, The Northern Gentry and the Great Rebellion, in: Northern History 15 (1979).

${ }^{23}$ Die beste Darstellung der yeomen bleibt Campbell, The English Yeoman.

${ }^{24}$ Für die Untersuchung einzelner Gemeinden, in denen diese Bestimmungen herausgearbeitet werden, s. zum Beispiel William George Hoskins, The Midland Peasant. The Economic and Social History of a Leicestershire Village (London 1957), bes. Kap. 6 und 7; Margaret Spufford, Contrasting Communities. English Villagers in the Sixteenth and Seventeenth Centuries (Cambridge 1974), vor allem Teil 1; David G. Hey, An English Rural Community. Myddle under the Tudors and Stuarts (Leicester 1974), bes. Kap. 3-5. Umfassender angelegte Untersuchungen der Unterschiede in Reichtum und Lebensstandard zwischen diesen Gruppen bei David Cressy, Literacy and the Social Order. Reading and Writing in Tudor and Stuart England (Cambridge 1980) 137-139; A. Everitt, Farm Labourers; Peter Bowden, Agricultural Prices, Farm Profits and Rents, in: Thirsk (Hg.), Agrarian History, bes. Teil C; Maurice Wr. Barley, Rural Housing in England, in: Thirsk (Hg.), Agrarian History, bes. Teile E-G; s. auch Wrightson, English Society, 31-36.

${ }^{25}$ Clark/Slack, English Towns in Transition, $120 \mathrm{f}$.; $R$. Grassby, Social Mobility and Business Enterprise in Seventeenth-Century England, in: Donald Pennington/Keith Thomas (Hg.), Puritans and Revolutionaries. Essays in Seventeenth-Century History Presented to Christopher Hill (Oxford 1978); Wilfrid R. Prest, The Inns of Court under Elizabeth I and the Early Stuarts 1590-1640 (London 1972) 26-31; Brian P. Levack, The Civil Lawyers in England 1603-1641 (Oxford 1973) 10f.; Peter Clark, The civic leaders of Gloucester 1580-1800, in: Peter Clark (Hg.), The Transformation of English Provincial Towns 1600-1800 (London 1984). 
züglich der Teilnahme am cursus bonorum des bürgerlichen Lebens, die es zwischen Handelsunternehmen und Handwerksbetrieben gab, zwischen unabhängigen Meistern und gegen Lohn arbeitenden „Gesellen“, freemen und den immer noch wenig erforschten Rängen der Tagelöhner und der städtischen Armen ${ }^{26}$.

In all diesen Bereichen haben sozialstrukturelle Untersuchungen unser Verständnis des Schichtungsprofils der Gesellschaft im 16. und 17.Jahrhundert verbessert. Neuere Untersuchungen gehen noch über die ursprüngliche Konzentration auf Reichtum, Ansehen und Amtsträgerschaft hinaus, um weitere Dimensionen sozialer Differenzierung zu erforschen. Immer wieder ist die traditionelle soziale Hierarchie mit signifikanten und gelegentlich unerwarteten Kombinationen von Status- und Berufsgruppen wiedergegeben worden: mit solch einem demographischen Merkmal wie dem des Heiratsalters ${ }^{27}$; mit der Struktur des Haushaltes und der geographischen Mobilitāt ${ }^{28}$; mit dem Verhalten und den Einstellungen der Familie ${ }^{29}$; mit den Bildungschancen

${ }^{26}$ Clark/Slack, English Towns in Transition, Kap. 8-9; Jobn Patten, English Towns 1500-1700 (Folkstone and Hamden, Conn., 1978) Kap. 4 \& 6; William George Hoskins, Provincial England. Essays in Social and Economic History (London 1965) Kap. 4; Julian Cornwall, English Country Towns in the Fifteen Twenties, in: Economic History Review, 2nd series, 15 (1962) $61 \mathrm{ff}$.; Alan D. Dyer, The City of Worcester in the Sixteenth Century (Leicester 1973) Kap. 14; C. PhytbianAdams, Desolation of a City. Coventry and the Urban Crisis of the Late Middle Ages (Cambridge 1979) Kap. 10; David M. Palliser, Tudor York (Oxford 1979) Kap.4-6; T. S. Willian, Elizabethan Manchester (Chetham Society, 3rd series, 27, 1980), Kap. 6; Vivian Brodsky Elliott, Mobility and Marriage in Pre-Industrial England (Unveröffentlichte Ph. D., University of Cambridge 1978) Teil 1; S. Rappaport, Social Structure and Mobility in Sixteenth Century London. Part 1, in: London Journal 9 (1983); J. P. Boulton, The Social and Economic Structure of Early SeventeenthCentury Southwark (Unveröffentlichte Ph. D., University of Cambridge 1983); D. V. Glass, Socioeconomic status and occupations in the City of London at the end of the seventeenth century, in: Peter Clark (Hg.), The Early Modern Town. A Reader (London 1976).

${ }^{27} R$. B. Outbwaite, Age at marriage in England from the late seventeenth to the nineteenth century, in: Transactions of the Royal Historical Society, 5th series, 23, 1973); Vivian Brodsky Elliott, Single Women in the London Marriage Market: Age, Status and Mobility 1598-1619, in: $R$. $B$. Outbwaite (Hg.), Marriage and Society. Studies in the Social History of Marriage (London 1981). Ein Versuch, demographische Unterschiede zwischen landbesitzenden und landlosen Familien festzustellen, bei Victor Skipp, Crisis and Development. An ecological case study of the Forest of Arden 1570-1674 (Cambridge 1978) Kap.4.

${ }^{28}$ Peter Laslett, Mean household size in England since the sixteenth century, in: Peter Laslett/Richard Wall $(\mathrm{Hg}$.), Household and family in past time (Cambridge 1972) 154; N. Goose, Household size and structure in early-Stuart Cambridge, in: Social History 5 (1980); Hey, An English Rural Community, 117, 141 f., 170, 173-76; Keith Wrigbtson/David Levine, Poverty and Piety in an English Village: Terling 1525-1700 (New York/San Francisco/London 1979) 81 f.; Peter Clark, Migration in England During the Late Seventeenth and Early Eighteenth Centuries, in: Past and Present 83 (1979).

${ }^{29}$ Auf soziale Unterschiede im Familienleben wird wiederholt hingewiesen bei Lawrence Stone, The Family, Sex and Marriage in England 1500-1800 (London 1977); Wrightson, English Society, Kap. 3 \& 4; R. A. Houlbrooke, The English Family 1450-1700 (London/New York 1984). 
und dem Grad der Alphabetisierung ${ }^{30}$; mit den Formen von Kriminalisierung ${ }^{31}$; mit den Einstellungen, den Sitten und Bräuchen sowie der Teilhabe an religiösen und politischen Bewegungen ${ }^{32}$.

Derartige Arbeiten haben deutlich den bestimmenden Einfluß aufgezeigt, den die relative soziale Position auf den Lebenslauf und die Chancen der Individuen ausübte. Mehr noch: Sie haben aufgedeckt, daß es regionale und lokale Abweichungen gab, sowohl was die Schichtungsstruktur angeht als auch hinsichtlich der Komplexität der Unterscheidungen zwischen sozialen Gruppen. Diese Abweichungen können auf eine plausible Art und Weise mit unterschiedlichen Wirtschafts- und Beschäftigungsstruk-

${ }^{30}$ David Cressy, Educational Opportunity in Tudor and Stuart England, in: History of Education Quarterly 16 (1976); ders., Literacy and the Social Order, bes. Kap.6; s. auch Spufford, Contrasting Communities, Teil 2; Wrightson/Levine, Poverty and Piety, 146-152; R. A. Houston, The Development of Literacy: Northern England 1640-1750, in: Economic History Review 35 (1982).

${ }^{31}$ Vorreiter einer Untersuchung der Verknüpfungen zwischen sozialem Status, Kriminalität, Verfolgung und Bestrafung war Joel Samaha, Law and Order in Historical Perspective. The Case of Elizabethan Essex (New York/London 1974). Seit der Veröffentlichung von James S. Cockburn, Early-Modern Assize Records as Historical Evidence, in: Journal of the Society of Archivists 5 (1975), sind Kriminalitätsforscher wesentlich vorsichtiger dabei geworden, den angegebenen Status angeklagter Personen zu übernehmen. Dennoch sind soziale Unterschiede bezüglich Verfolgung und Bestrafung in einer Reihe lokal begrenzter Untersuchungen nachgewiesen worden, und sie werden im allgemeinen durch breiter angelegte Kriminalitätsstudien bestätigt. S. z. B. James S. Cockburn, The Nature and Incidence of Crime in England 1559-1625. A Preliminary Survey, in: ders., Crime in England 1550-1800 (London 1977) 61-64; M. J. Ingram, Communities and Courts. Law and Disorder in Early Seventeenth-Century Wiltshire, in: ebd., 129-34; Wrightson/Levine, Poverty and Piety, Kap. 5; J. A. Sharpe, Crime in Early Modern England 1550-1750 (London/New York 1984).

${ }^{32}$ Der Bereich „kultureller Unterschiede" tritt deutlich hervor in Arbeiten wie Christopher Hill, Society and Puritanism in Pre-revolutionary England (London 1964); Thomas, Religion and the Decline of Magic; ders., Man and the Natural World. Changing Attitudes in England 1500-1800 (London 1983); Keith Wrightson, Alehouses, Order and Reformation in Rural England 1590-1660, in: Eileen Yeo/Stephen Yeo (Hg.), Popular Culture and Class Conflict 1590-1914. Explorations in the History of Labour and Leisure (Brighton 1981); ders., Two concepts of order: justices, constables and jurymen in seventeenth century England, in: Jobn Brewer/Jobn Styles (Hg.), An Ungovernable People. The English and their law in the seventeenth and eighteenth centuries (London 1980). Untersuchungen in diesen Bereichen können selten quantifizierend betrieben werden, obwohl es einige Versuche gibt, soziale Unterschiede in bezug auf die Neigung zu religiöser Nonkonformität zu quantifizieren. S. zum Beispiel Spufford, Contrasting Communities, 298-306; J. J. Hurwich, Dissent and Catholicism in English Society. A Study of Warwickshire 1660-1720, in: Journal of British Studies 16 (1976); Wrightson/Levine, Poverty and Piety, 124-33, 154-72 und Kap.7; Richard T. Vann, The Social Development of English Quakerism 1655-1755 (Cambridge, Mass., 1969) Kap. 2; B. Reay, The Social Origins of Early Quakerism, in: Journal of Interdisciplinary History 11 (1980); Brian S. Capp, The Fifth Monarchy Men. A Study in Seventeenth-Century English Millenarianism (London 1972) Kap.4; J. F. McGregor/B. Reay (Hg.), Radical Religion in the English Revolution (Oxford 1984) 18f., 29, 35-39, 143 f., 173. Eine Diskussion politischer Teilhabe unterhalb der Ebene der gentry und der städtischen Eliten bei Derek Hirst, The Representative of the People? Voters and Voting in England under the Early Stuarts (Cambridge 1975); Brian Manning, The English People and the English Revolution 1640-49 (London 1976); David Underdown, The Problem of the Popular Allegiance in the English Civil War, in: Transactions of the Royal Historical Society, 5th series, 31 (1981). 
turen verknüpft werden, mit dem Einfluß eines bestimmten lokalen Herkommens (etwa der Erbfolge) und mit unterschiedlicher Einbindung in Prozesse ökonomischen, sozialen und kulturellen Wandels. Es gab keine statische, „traditionale“ Sozialordnung, sondern eine dynamische Gesellschaft, in der sich im Laufe der Zeit sowohl das Schichtungsprofil als auch die Erfahrung einzelner Gruppen veränderten. Aus der Untersuchung solcher Veränderungen wissen wir um Langzeit-Entwicklungen, die die Zeitgenossen nur undeutlich spürten: Verschiebungen der sozialen Verteilung von Grund und Boden sowie des Reichtums; zahlenmäßige Zunahme der gentry sowie der akademischen und der handeltreibenden „Mittelschicht“; der Niedergang der wenig Land besitzenden „husbandmen" auf dem flachen Land; die Ausdehnung industrieller Beschäftigung; der massive Zuwachs der "labouring poor"; die relative Festschreibung oder Verminderung von Lebensstandards; das Auftauchen neuer Unterscheidungen in der Erziehung, in den Einstellungen und im Verhalten ${ }^{33}$.

Wir haben allen Grund, den Autoren der sozialstrukturellen Studien wegen dieser Erkenntnisse dankbar zu sein. Die Leistungen der neueren englischen Sozialgeschichte bei der Rekonstruktion der Strukturen und Triebkräfte jener Welt, die Peter Laslett 1965 als "The world we have lost" bezeichnete, beruhen zum großen Teil auf „sozial-spezifischen“ Analysen. Aus genau diesem Grund sollten wir uns aber auch daran erinnern, daß auch diese Arbeiten ihre Grenzen haben. Selbst wenn eine quantifizierende Analyse möglich ist, liefert die begeisterte Produktion sauberer tabellarischer Hierarchien auf der Basis einer Vielzahl von Schichtungskriterien bestenfalls unvollstāndige Hinweise auf die zentralen Realitäten der Sozialordnung. Derartige analytische Konstruktionen darf man nicht zu wörtlich nehmen, weil sie sonst die Aufmerksamkeit von manchen Komplexitäten und Uneindeutigkeiten ablenken.

Bei den Oberbegriffen scheint es klar genug zu sein, daß die traditionellen Kategorien der "degrees“ und „callings“ deutliche Hinweise auf den relativen Wohlstand, den sozialen Rang und die Chancen der jeweiligen Individuen geben. Es muß aber betont werden, daß solche sauberen Einteilungen scheitern oder sich zumindest an ihren Rändern auflösen, wenn man sie im Detail untersucht. Soziale Zuordnungen auf der Grundlage dieser Kategorien zeigen immer wieder zweierlei: Einerseits gibt es deutlich erkennbare Unterschiede, was den Durchschnitt betrifft. Andererseits gibt es auch sehr beträchtliche Überschneidungen zwischen benachbarten Sozialkategorien ${ }^{34}$. Das allein sollte genügen, uns daran zu erinnern, daß die "degrees“ und "callings“, wie sie die Zeitgenossen unterschieden und auflisteten, bei weitem keine exakt definierten, wohlunterscheidbaren oder homogenen sozialen Gruppen bildeten ${ }^{35}$. Das Aufstellen

\footnotetext{
${ }^{33}$ Ausführlicher und mit weiteren Nachweisen Wrightson, English Society, bes. Kap. 2 und 5-7. ${ }^{34}$ Für Überschneidungen in der Verteilung von Reichtum s. z.B. M. Brigg, The Forest of Pendle in the seventeenth century, in: Transactions of the Historic Society of Lancashire and Cheshire 115 (1962); P. Tyler, The status of the Elizabethan parochial clergy, in: G. J. Cuming ( $\mathrm{Hg}$.), Studies in Church History IV: The Province of York (Leiden 1967) 96f.; Spufford, Contrasting Communities, 36-41. Solche Úberschneidungen sind zum Beispiel ebenso deutlich in det sozialen Verteilung des Analphabetentums oder der Neigung zu religiösem Nonkonformismus zu finden. ${ }^{35}$ Dies wird sehr entschieden vertreten bei $G$. A. Kerby, Inequality in a Pre-Industrial Society (Unveröffentlichte Ph. D., University of Cambridge 1983) Kap.9.
} 
von Tabellen vermag den falschen Eindruck einer Eindeutigkeit sozialer Einteilung zu erwecken. Das aber verbirgt nur den komplexen Charakter des Prozesses sozialer Einschätzung.

Zweitens wird die Verwendung traditioneller Einteilungen praktisch sinnlos, wenn wir uns mit der Einordnung bestimmter Berufsgruppen mit unklarem Status in den zeitgenössischen Gesellschaftsbeschreibungen beschäftigen. Kaufleute und Händler, Rechtsanwälte, der Klerus, ländliche und städtische Handwerker können aus einem einfachen Grund nicht ohne weiteres in die Standard-Hierarchie eingeordnet werden: Es handelt sich nicht um einheitliche oder homogene Gruppen. Eine soziale Schichtung, die als einziges Kriterium „Handwerk und Handel“ verwendet, verbirgt wahrscheinlich mehr, als sie enthüllt. Zweifellos gab es in bestimmten Stādten eine klare Rangfolge innerhalb von Handel und Handwerk. Auch auf dem Land waren Händler und Handwerker deutlich geschichtet. Einige waren nach Lebensführung und Stellung mit wohlhabenden yeomen vergleichbar. Andere gehörten mehr zu den Landarbeitern. Viele lebten von zwei oder mehr Beschäftigungen, was solche Unterscheidungen bedeutungslos werden läßt ${ }^{36}$.

Drittens: Nimmt man Schichtungsstudien zu wörtlich, kann die Aufmerksamkeit für die dauernde individuelle soziale Mobilität nachlassen. Wie wir gesehen haben, waren die Zeitgenossen bereit, die Tatsache solcher Möglichkeiten anzuerkennen. Einzelfallstudien bestätigen dies. Wir besitzen zahllose Übersichten über gentry-Familien in bestimmten Grafschaften und deren großes oder geringes Alter. Während wir aber mehr und mehr über soziale Schichtung im frühneuzeitlichen England wissen, wissen wir immer noch zu wenig über die Möglichkeiten relativer sozialer Mobilität und ihre mögliche Bedeutung für eine Abschwächung der Abgrenzung zwischen bestimmten, traditionell getrennten Gruppen oder für die Verhärtung der Grenzen zwischen anderen $^{37}$.

${ }^{36}$ Für die Städte s. o. Anm. 25. Für das Land s. zum Beispiel Hoskins, Midland Peasant, $166 \mathrm{f}$.; Wrightson/Levine, Poverty and Piety, $22 \mathrm{f}$.; Brigg, The Forest of Pendle, 88-90; David Hey, The Rural Metalworkers of the Sheffield Region. A Study of Rural Industry before the Industrial Revolution (Occasional Papers of the Department of Local History, 2nd series No. 5, Leicester 1972) 19-31, 34; G. D. Ramsay, The Wiltshire Woollen Industry in the Sixteenth and Seventeenth Centuries (London ${ }^{2} 1965$ ) Kap. 2 \& 3; Joan Thirsk, Industries in the countryside, in: Frederick J. Fisher $(\mathrm{Hg}$.), Essays in the Economic and Social History of Tudor and Stuart England (Cambridge 1961); Marie Rowlands, Masters and Men in the West Midland metalware trades before the industrial revolution (Manchester 1975) Kap. 1-3, 6.

${ }^{37}$ Eine allgemeine Diskussion der Probleme sozialer Mobilität bei Stone, Social Mobility in England; Alan Everitt, Social Mobility in Early Modern England, in: Past and Present 33 (1966); Peter Laslett, The World We Have Lost further explored (London 1984) $238 \mathrm{ff}$. Versuche, das tatsāchliche Ausmaß sozialer Mobilität zu bestimmen, gibt es viel zu wenige, hauptsächlich weil es Schwierigkeiten bereitet, ein Sample von Individuen über einen gewissen Zeitraum zu verfolgen. S. Zum Beispiel Cliffe, The Yorkshire Gentry, 16; Blackwood, The Lancashire Gentry, $18 \mathrm{fft}$ und Appendix I; Brodsky Elliott, Mobility and Marriage, Teil III, Kap. 3; Wrightson/Levine, Poverty and Piety, 106-108; Lawrence Stone/J. C. F. Stone, An Open Elite? England 1540-1820 (Oxford 1984). Aspekte sozialer Mobilitāt in Verbindung mit bestimmten Lebensphasen werden betont bei Hoskins, Midland Peasent, 147; Brigg, The Forest of Pendle, 74f.; Hey, An English Rural Community, $170 \mathrm{ff}$; Paul Slack, Social problems and social policies, in: The Traditional Com- 
Schließlich gibt es das Problem, daß die Bedeutung traditioneller Statusbezeichnungen sich von Ort zu Ort oder über einen bestimmten Zeitraum ändern konnte. Wenn wir gentleman oder yeoman aus einer bestimmten Zeit und Gegend und gentleman oder yeoman aus einer anderen Zeit und Gegend gegenüberstellen, vergleichen wir möglicherweise nicht Gleiches mit Gleichem. Im Nordosten Englands zum Beispiel wurde der Begriff yeoman während der gesamten Frühen Neuzeit unterschiedslos zur Bezeichnung kleiner Landbesitzer benutzt. Er trug nicht die im Süden gelāufige Bedeutung eines höheren sozialen Status. Obwohl die Veränderungen der Status-Bezeichnungen noch nicht hinreichend erforscht sind, ist es allgemein bekannt, daß der Begriff gentleman um 1700 viel großzügiger benutzt wurde als im 16. Jahrhundert, wohingegen andere Begriffe, wie yeoman oder husbandman, überflüssig wurden ${ }^{38}$.

Kurz: Neuere Versuche, die traditionelle Hierarchie mit der sozialen Schichtung von Reichtum, mit der Qualifizierung als Amtsinhaber, mit dem Grad der Alphabetisierung usw. zu verknüpfen, haben zweifellos den realen Gehalt einiger feinsinniger Differenzierungen der Zeitgenossen nachgewiesen. Aber wenn wir die wertvollen Nachweise solcher Untersuchungen betrachten, müssen wir unsere Aufmerksamkeit auch mehr als üblich den Minderheiten zuwenden, den Ausnahmen, den Fällen, die nicht passen, den Kategorien, die sich überschneiden oder die sich bei näherer Untersuchung auflösen, die ihre Bedeutung von Ort zu Ort und von Zeit zu Zeit wechseln oder die durch die Möglichkeit sozialer Mobilität verwischt werden konnten. Die vertrauten Titel und die Reihenfolge, in die sie herkömmlicherweise gestellt wurden, waren bis zu einem gewissen Punkt brauchbar. Sicherlich werden die englischen Historiker sie weiterhin benutzen. Aber sie sollten vorsichtig gehandhabt werden, vorzugsweise innerhalb eines bekannten lokalen Rahmens, in dem Quellen so miteinander verknüpft werden können, daß sie sich gegenseitig aufhellen und anderenfalls verborgene Bedeutungen zutage treten. Und wir müssen uns dauernd bewußt sein, daß diese Kategorien äußerst ungenau definiert waren, daß sie an den Rändern verschwammen und daß die Trennlinien durchlässig waren.

Dies führt zu einem letzten Punkt hinsichtlich der Schichtungsstudien. Ich habe bereits darauf hingewiesen, daß es mindestens zwei Arten gab, die Sozialordnung des frühneuzeitlichen Englands zu erfassen: nämlich in der kleinschrittig gegliederten Hierarchie formaler Konventionen und in Begriffen mit gröberen Unterscheidungen zwischen zwei oder drei großen Gruppen. Einer der wertvollsten indirekten Beiträge der sozialstrukturellen Untersuchungen liegt darin, daß sie diese Doppel- oder Mehr-

munity Under Stress, Open University Course A322, English Urban History 1500-1780, Book III (Milton Keynes 1977) 84. S. auch bezüglich Armut und Lebensphasen den neueren Beitrag von W. Newman Brown und T. C. Wales zu R. M. Smith (Hg.), Land, Kinship and Lifecycle (Cambridge 1984).

${ }^{38}$ Cressy, Literacy and the social order, 125; A. Everitt, Change in the Provinces. The Seventeenth Century (Occasional Papers of the Department of Local History, 2nd series, No. 1, Leicester 1970) $43 \mathrm{ff}$.; P. Styles, The social structure of Kineton Hundred in the Reign of Charles II, in: Essays in honour of Pbilip B. Chatwin. Transactions and Proceedings of the Birmingham Archaeological Society 78 (1962) 102 f.; Geoffrey Holmes, Augustan England. Professions, State and Society 1680-1730 (London 1982) 9, 11; William A. Speck, Stability and Strife. England 1714-1760 (London 1977) 37, 40f., 47 f. 
facherfassung klarer gemacht haben. Zwar haben sie gezeigt, daß die traditionelle Hierarchie in vielen Lebensbereichen reproduziert wurde, sie haben aber auch gezeigt, 1), daß man die Brüche, die im Schichtungsprofil aufgezeigt werden können, nicht einfach wieder in die traditionellen „degrees“ einbetten kann, und 2), daß diese Brüche sich in ihrer sozialen Bedeutung und Reichweite unterschieden.

Daraus folgt, daß man von bestimmten Status- und Berufsgruppen sagen kann, sie bildeten einen „cluster" oder "Gruppierungen“, in denen die soziale Distanz zwischen den Mitgliedern zwar vorhanden, aber nicht so tief und einschneidend war wie diejenige, die sie von anderen sozialen Gruppen trennte. So reichten die führenden städtischen Kaufleute und die höheren akademischen Ränge an die landbesitzende gentry heran, was ihre Geburt, die Bildung, das Einkommen, die Lebensführung und die Teilhabe an der Macht betraf. Ein anderes Beispiel: Kleinbäuerliche husbandmen auf dem Dorf mögen in ihrem Lebensstandard, ihren Sitten, ihrem Analphabetentum und der Struktur ihrer Haushalte dem Kötter und dem Landarbeiter näher gestanden haben als ihrem wohlhabenderen yeoman-Nachbarn, der Amtsträger war, sich Diener hielt und häufig lesen konnte. Yeomen wiederum hatten viel gemein mit der niederen gentry und der städtischen „Mittelschicht“.

Solche Verwandtschaften oder Gruppierungen werden durch die allmählich anwachsenden Kenntnisse sozialer Differenzierungen und der Möglichkeit sozialer Mobilität sehr nahe gelegt. Neuerdings sind sie in überzeugender Art und Weise durch Vivian Brodsky Elliott bestätigt worden. In einer bemerkenswert originellen Arbeit hat sie gezeigt, daß es möglich ist, die relative Distanz zwischen Status- und Berufsgruppen zu bestimmen, indem man die von Individuen und Familien getroffene Auswahl von Ehepartnern und die Lehrstellen der Söhne systematisch untersucht. Ihre Ergebnisse führen nicbt zu einer gleichmäßig abgestuften Hierarchie, sondern zu einer Anzahl von Gruppierungen aus Status- und Berufsgruppen, deren jeweilige Nähe sich festmachen läßt an der Bereitschaft, untereinander zu heiraten, und an der Übereinstimmung bei der Art, ihre Söhne in städtischen Unternehmungen unterzubringen ${ }^{39}$.

Aus all dem läßt sich entnehmen, daß es innerhalb der vielfältigen Hierarchie formaler Gesellschaftsbeschreibung etwas gab, was man vielleicht als soziales und kulturelles Milieu bezeichnen könnte. Vielleicht versuchten die Zeitgenossen, derartige Fügungen von sozialen Gruppen zu erfassen, wenn sie Bezeichnungen wie "gentlemen“, "the quality", „the best inhabitants“, "the middling sort", „the better sort", „the meaner sort", "the inferior sort" und viele andere informelle, ungenaue, aber gesellschaftlich zutreffende Einschätzungen benutzten. Hier zeigte sich eine Alternative bei der Betrachtung gesellschaftlicher Strukturen, die mit den feineren Unterscheidungen der vollständigen sozialen Hierarchie keineswegs unvereinbar war. Sie berief sich aber weniger auf statische, formalisierte Unterscheidungen, als vielmehr auf grundlegende Einteilungen, wie sie in der Dynamik der alltäglichen sozialen Beziehungen deutlich wurden. Mehr noch: Wenn diese Alternative die vorherrschende Form der Wahrnehmung wurde, wie ich vorgeschlagen habe, dann hing ihre zunehmende Bedeutung möglicherweise mit den sozialen Veränderungen des späten 16. und des 17.Jahrhun-

${ }^{39}$ Brodsky Elliott, Mobility and Marriage, Teil I, Kap. 2-4. 
derts zusammen: mit der Zunahme der niederen gentry; dem Auftauchen der städtischen „pseudo gentry“; der mannigfaltiger werdenden, erfolgreichen „Mittelschicht“ in Stadt und Land; dem allmählichen Verschwinden des ackerbautreibenden husbandman; dem Anwachsen der labouring poor; mit den komplexen Prozessen ökonomischen, politischen und kulturellen Wandels, die eine stärker integrierte Gesellschaft auf nationaler Ebene und eine stärker aufgefächerte und gegliederte lokale Gesellschaft zur Folge hatten ${ }^{40}$.

\section{Ergebnisse}

Zusammengenommen liefern die beiden Möglichkeiten, die Sozialstruktur Englands im 16. und 17. Jahrhundert zu rekonstruieren, ein einigermaßen deutliches Bild. Es war eine im höchsten Maß gegliederte Gesellschaft, aber die Konstellationen der Ungleichheit waren alles andere als einheitlich, rigid oder unveränderlich. Die Kriterien sozialer Einschätzung waren komplex und ungenau bestimmt, gleichwohl war Reichtum ein entscheidender Faktor. Individuelle soziale Mobilität gab es dauernd, und sie wurde offen vermerkt. Es gab betrāchtliche Verānderungen sowohl der relativen Größe bestimmter sozialer Gruppen als auch des Charakters und des Ausmaßes ihrer Unterschiede. In der Alltagssprache und im täglichen Umgang wurde das traditionelle Bild der sozialen Hierarchie in eine einfachere, weniger formale Klassifikation aufgelöst, in der der soziale Abstand auf der Grundlage unterschiedlichster Kriterien zu einer Reihung von Gruppierungen führte, die jeweils unterscheidbare soziale und kulturelle Milieus darstellten. Es gab mindestens zwei Möglichkeiten, die Sozialstruktur zu verstehen. Beide gaben gleichermaßen gültige, aber alternative Auffassungen einer komplexen Wirklichkeit wieder, und die eine verdrängte allmählich die andere. Das Reden von „degrees“ wurde ein Anachronismus, wurde zur Vokabel eines sozialen Konservativismus. Das Reden von „sorts“ traf die soziale Realität immer besser, weil es einer sich entwickelnden Strukturveränderung in der englischen Gesellschaft besser Ausdruck verleihen konnte. Der Charakter des Übergangs in der sozialen Wirklichkeit und in deren Wahrnehmung wird vielleicht in einer Antwort Gregory Kings verkörpert, die er 1697 einem Kritiker der in seiner berühmten Sozialstatistik enthaltenen Berechnungen gab. Innerhalb eines einzigen Abschnittes, in dem King seine Methodologie erläuterte, bezog er sich zunächst auf die „several degrees“ des englischen Volkes; dann auf: "the better sort", "the middle sort" und "the poorest sort"; und schließlich entschied er sich, die Bevölkerung in "classes“ einzuteilen ${ }^{41}$. Hier handelt es sich um eines der frühesten Beispiele einer neuen Art der Gesellschaftsbeschreibung, um die Verwendung des Begriffes „class“, die im Verlauf des 18. Jahrhunderts an Bedeutung gewann und die heute noch verbreitet ist.

${ }^{40}$ Diese Entwicklungen werden umfassend diskutiert bei Wrightson, English Society, Kap. 5-7.

${ }_{11}$ Thirsk/Cooper, Seventeenth-Century Economic Documents, 795. Für das allmähliche Auftauchen des "class"-Begriffes seit dem 18.Jahrhundert s. Asa Briggs, The Language of "Class" in Early Nineteenth-century England, in: Asa Briggs/Jobn Saville ( $\mathrm{Hg}$.), Eassays in Labour History. In memory of G. D. H. Cole (London 1960). 
